\title{
3 Anfänge der Kinematographie in Österreich
}

\subsection{Vorführkonzeptionen und Programmgestaltung}

Nachdem die Brüder Lumière am 28. Dezember 1895 am Boulevard des Capucines in Paris die kinematographische Technik erstmals in einem öffentlich-kommerziellen Rahmen vorgeführt hatten, folgten im Februar des Folgejahres Präsentationen in London, Bordeaux und Brüssel. Am 20. März 1896 wurden die „lebend Photographien“ schließlich auch in Wien vorgestellt. Erste private Vorführungen fanden in der k.k. Graphischen Lehr- und Versuchsanstalt statt. Die öffentliche Premiere vollzog sich am gleichen Tag im ersten Wiener Gemeindebezirk, Kärntner Straße 45/Ecke Krugerstraße. Für 50 Kreuzer wurde ein Nonstop-Programm von 10 Uhr früh bis 8 Uhr abends angeboten. ${ }^{91}$ Am 27. März gab man in der Französischen Botschaft sowie im Haus des Niederösterreichischen Gewerbevereins weitere Interessentenvorstellungen. Bis Ende 1896 wurde der Kinematograph der Brüder Lumière in allen großen Städten der Monarchie in Wanderkinounternehmungen präsentiert. ${ }^{92}$

Varietés zählten schon vor der Jahrhundertwende, mobile Kinoeinrichtungen noch bis $1910 \mathrm{zu}$ den dominanten Film-Vorführungsorten Österreich-Ungarns. ${ }^{93}$ Die Filmknappheit der Pionierzeit verlangte ein variantenreiches Hauptprogramm bzw. einen raschen Ortswechsel. Zu schnell erschöpfte sich vor allem in Kleinstädten das Publikumsinteresse an den oftmals nur 10 bis 15 Minuten dauernden kinematographischen Darbietungen. Die meisten Laufbilder wurden von kleingewerblichen Produzenten in geringen Mengen für den Eigenbedarf erzeugt. Da es an einem ausgebildeten Verleihsystem mangelte, wurden die Filme direkt beim Erzeuger gekauft oder unter den Wanderunternehmern getauscht. Die erste professionelle Filmvertriebsorganisation der Monarchie lässt sich 1905 in Wien ausmachen. ${ }^{94}$

91 Die Autorin zitiert hier Kapitel 2 ihres Artikels: Moser, Karin: „Frühes Kino“ - Attraktion und Erlebniswelt. Die Entwicklung eines Mediums, Kapitel 2 „Der erste Kinematograph in Wien - ein Medium erobert Österreich-Ungarn“, in: Virtuelle Ausstellung „Der Erste Weltkrieg und das Ende der Habsburgermonarchie, http://ww1.habsburger.net/de/kapitel/der-erste-kinematograph-wienein-medium-erobert-oesterreich-ungarn, 30.11.2014.

92 Vgl. Kieninger, Ernst: Das „klassische Wanderkino“ 1896-1914. Filmkommunikation auf dem Weg zur Institution am Beispiel Niederösterreich und Umland, Dipl., Wien 1992, S. 56. Fritz, Walter: Im Kino erlebe ich die Welt. 100 Jahre Kino und Film in Österreich, Wien/München 1997, S. 11-14. Photographische Korrespondenz, Nr. 431, 1896, S. 31.

93 Schwarz, Werner Michael: Kino und Kinos in Wien. Eine Entwicklungsgeschichte bis 1934, Wien 1992, S. 17-19. Die Autorin zitiert hier Kapitel 3 ihres Artikels: Moser, „Frühes Kino“, Kapitel 3 „Vom Schaustellerbetrieb zum Kinotheater“, http://ww1.habsburger.net/de/kapitel/vomschaustellerbetrieb-zum-kinotheater, 30.11.2014.

94 Kieninger, Wanderkino, S. 74, 78, 115. Schwarz, Kino und Kinos, S. 19. 
Das damals international ausgerichtete Filmprogramm dominierten französische Hersteller. ${ }^{95}$ In der Periode 1896 bis 1914 bezogen die Wanderkino-unternehmer ihre Lichtbilder zu 67,5 Prozent bei der Firma „Pathé“. Mit „Raleigh \& Roberts“ (6,1 Prozent), „Éclipse“ (4,3 Prozent), „Gaumont“ (3,5 Prozent) und „Lumière“ (2,3 Prozent) fanden sich weitere französische Unternehmen unter den Hauptlieferanten. Erst danach folgte mit der Deutschen „Bioskop“ (2,1 Prozent) ein anders verorteter Handelspartner. Ein Anteil von rund 2 Prozent wurde von den mobilen Kinobetreibern selbst gedreht. $^{96}$

Das Kino der Frühzeit stand dem Varieté bedeutend näher als dem Theater. ${ }^{97}$ Die Vielfalt der Attraktionen bestimmte auch den Film der Pionierzeit. Dieser fand auf Festen und Jahrmärkten, wo sich die kinematographischen Schausteller vornehmlich einfanden, ${ }^{98}$ einen stimmigen Präsentationsrahmen. Die filmischen Vorführungen waren oftmals Teil einer abwechslungsreichen Revue, die etwa „Abnormitäten“, Wachsfiguren, automatische Apparaturen, Kaiserpanoramen sowie Sketchs, artistische Darbietungen oder Tanz- und Gesangseinlagen präsentierte. ${ }^{99}$ Eine besondere Rolle kam den Rezitatoren zu, die das Geschehen auf der Leinwand dem filmisch nicht geschulten Publikum erläuterten, Orientierungspunkte gaben, das Gezeigte verorteten und räumliche Wechsel begründeten. Vortragskünstler und Musikbegleitung dienten als Korrektiv zur fehlenden Filmsprache. Sie vermittelten eine spezielle Lesart der Bilder und passten den Inhalt den lokalen Gegebenheiten, dem Geschmack und den Interessen der jeweiligen Zuschauer an. ${ }^{100}$ Mit Einführung der Zwischentitel und dem allmählichen Einsatz interpretierender filmischer Mittel verlor das begleitende gesprochene Wort um 1910 seine Bedeutung. ${ }^{101}$ Im nicht kommerziellen Kino, und vor allem bei der

95 Die Autorin zitiert hier Kapitel 7 ihres Artikels: Moser, „Frühes Kino“, Kapitel 7 „Österreichische Filmpioniere“, http://ww1.habsburger.net/de/kapitel/oesterreichische-filmpioniere, 30.11.2014.

96 Die Zahlen wurden in einer umfassenden Studie über Wanderkinobetriebe in Niederösterreich und seinem Umland von Ernst Kieninger erhoben und lassen durchaus Rückschlüsse auf das Gesamtgebiet Österreich-Ungarns zu. Weitere Lieferanten waren zudem die britischen Unternehmen „Warwick und Urban“ sowie die deutsche Produktion „Messter“. Vgl.: Kieninger, Wanderkinos, S. 147, 317. 97 Die Autorin zitiert hier Kapitel 4 ihres Artikels: Moser, „Frühes Kino“, Kapitel 4 „Hereinspaziert! - Aufführungspraxen, Attraktionen, Schau- und Hörerlebnisse, http://ww1.habsburger.net/ de/kapitel/hereinspaziert-auffuehrungspraxen-attraktionen-schau-und-hoererlebnisse, 30.11.2014.

98 Warstat, Dieter Helmuth: Frühes Kino der Kleinstadt, Berlin 1982, S. 21-23

99 Kieninger, Wanderkinos, S. 61, 274.

100 Altenloh, Emilie: Soziologie des Kinos. Die Kinounternehmung und die sozialen Schichten ihrer Besucher, Jena 1914, S. 20. Châteauvert, Jean: Das Kino im Stimmbruch, in: KINtop5, „Aufführungsgeschichten“, Jahrbuch zu Erforschung des frühen Films, Basel/Frankfurt am Main 1996, S. 86-88. Gunning, Tom: Vor dem Dokumentarfilm. Frühe non-fiction-Filme und die Ästhetik der „Ansicht“, in: KINtop4, „Anfänge des dokumentarischen Films, Jahrbuch zur Erforschung des frühen Films, Basel/Frankfurt am Main 1995, S. 118.

101 Schwarz, Kino und Kinos, S. 89. 
Präsentation von Gebrauchsfilmen, zu denen auch der Industrie(werbe)film zählt, waren von Sprechern vorgetragene Begleittexte allerdings durchaus bis in die 1950er- und 1960er-Jahre üblich. ${ }^{102}$

Das dem Schaustellergewerbe entsprechende „Prinzip der Attraktion“ fand seine Analogie im Filmprogramm. Die bis 1906 dominierenden Einakter, die zudem fast ausschließlich in einer einzelnen Einstellung gedreht waren, ${ }^{103}$ wurden dramaturgisch in das Gesamtkonzept eingebaut und kurzweilig arrangiert. In den dargebotenen Nummernprogrammen ${ }^{104}$ wechselten die Wanderkinounternehmer (mitunter nachgestellte) Aktualitäten mit technischen, naturwissenschaftlichen Aufnahmen, Reise- und Landschaftsbildern oder „komisch-pikanten“ Filmen geschickt ab. Bei der Gestaltung der Filme und deren öffentlichen Inszenierung orientierten sich die Filmpioniere und Schausteller durchwegs an bereits etablierten Medien wie illustrierten Magazinen, Romanheften, Bildgeschichten, Postkarten, dem populären Theater, den Kaiserpanoramen und der Projektionskunst. ${ }^{105}$

\subsection{Die „Ästhetik der Ansicht“: „Naturaufnahmen“ als Vorläufer des werbenden Films}

In der Frühzeit der Kinematographie bestimmten Non-fiction-Filme das Nummernprogramm der Schausteller, wobei zwei Formen, die unter dem zeitgenössischen Begriff „Naturaufnahmen“"106 subsumiert wurden, am stärksten vertreten waren: Reisebilder, die Städte, Landschaften und exotische Gebräuche in den Fokus nahmen, und Industriebilder, die Warenfertigungs- und Arbeitsprozesse veranschaulichten. ${ }^{107}$ Diese frühen Filme zeugen von einer offensichtlichen voyeuristisch-exhibitionistischen

102 Vgl. dazu Zimmermann, Yvonne: Dokumentarischer Film: Auftragsfilm und Gebrauchsfilm, in: Zimmermann, Yvonne (Hg.): Schaufenster Schweiz. Dokumentarische Gebrauchsfilme 1896-1964, Zürich 2011, S. $81 \mathrm{f}$.

103 Gunning, Tom: The Non-Continous Style of Early Film (1900-1906), in: Österreichische Gesellschaft für Filmwissenschaft, Kommunikations- und Medienforschung (Hg.): Arbeitsmappe. Filmstatistik und Dokumentation“, Wien 1983, S. 1.

104 Die Autorin zitiert hier Kapitel 5 ihres Artikels: Moser, „Frühes Kino“, Kapitel 5 „Nummernprogramme - Kleinode aus aller Welt, http://ww1.habsburger.net/de/kapitel/nummernprogrammekleinode-aus-aller-welt, 30.11.2014.

105 Garncarz, Joseph: Nicht-fiktionale Filmformen in Varietés und Wanderkinos, in: Jung, Uli/Loiperdinger, Martin (Hg.): Geschichte des dokumentarischen Films in Deutschland, Bd. 1, Kaiserreich 1895-1918, Stuttgart 2005, S. 74-76. Kieninger, Wanderkinos, S. 130, 274 und 309.

106 Im Fachkontext der Zeit wurde wiederholt auf Bilder, welche „die Vorgänge nach der Natur widerspiegeln“ verwiesen. Vgl. Garncarz, Joseph: Der nicht-fiktionale Film im Programm der Wanderkinos, in: Jung, Uli/Loiperdinger, Martin (Hg.): Geschichte des dokumentarischen Films in Deutschland, Bd. 1, Kaiserreich 1895-1918, Stuttgart 2005, S. 117.

107 Ebd., S. 116-118. 
Übereinkunft: Das Beobachten wie auch das Zurschaustellen sind am Filmmaterial klar nachvollziehbar. Landschaften werden von erhöhten Standpunkten überblickt oder mit der Kamera abgeschwenkt. Nah- und Detailaufnahmen machen Arbeitsvorgänge genau nachvollziehbar. Werktätige oder Passanten werden abgelichtet, während sie sich vor der Kamera inszenieren, Blicke austauschen, den Akt des Gefilmt-Werdens fortlaufend thematisieren und bildlich umsetzen. ${ }^{108}$

Der Filmwissenschaftler Tom Gunning prägte für diese Art des vordokumentarischen Films den Begriff „Ansicht“, womit er sich an die (etwa im Katalog der Brüder Lumière) im Zeitkontext gebräuchlichen Bezeichnungen „vue“ oder „view“ anlehnte. Den so benannten Aufnahmen fehlte eine zusammenhängende Erzählung ${ }^{109}$; einzelne Episoden wurden ohne narrative Abfolge aneinandergereiht. Man zeigte, beobachtete, erforschte das Ersichtliche, ohne eine eindeutige Interpretation und Argumentation einzubringen. ${ }^{110}$ Damit unterscheidet sich die „Ansicht“ vom Dokumentarfilm, der nicht nur beschreibt, sondern durch das gezielte Anordnen (Schnitt, Montage, Zwischentitel etc.) und die kreative Gestaltung des Materials eine dramaturgische Struktur vorgibt und einen Diskurs verfolgt. ${ }^{111}$

Arrangierte „Städteansichten“ zählten oftmals zu den ersten Bildern, die im Vorfeld kinematographischer Premieren gedreht und vorgeführt wurden. Die ansässige Bevölkerung sollte über die erste filmische Selbstpräsentation für das neue Medium gewonnen werden. Vor Ort gedrehte Lokalaufnahmen wurden von den Schaustellern in das Standardprogramm aufgenommen, mitunter sogar auf Wunsch des Mutterunternehmens - so etwa im Fall der Vertragspartner der Lumière'schen Kinematographenbetriebe.

Im April 1896 trafen in diesem Sinne im Auftrag der Firma Lumière der Operateur Alexander Promio und sein Gehilfe und Dolmetscher Alexander Werschinger in Wien ein, um folgende Aufnahmen auf Zelluloid zu bannen: DER STEPHANSDOM, DER STADTPark, Der TÜrkenschanzPark, Der Volksprater, Der Prater, Die HauptalleE, AusfahrT DER Wiener Fiaker, Korso DER SPAZIERgänger, Die DeutschmeisterkaPelle und DAS

108 Die Autorin zitiert hier Kapitel 5 ihres Artikels: Moser, „Frühes Kino“, Kapitel 5 „Nummernprogramme - Kleinode aus aller Welt, http://ww1.habsburger.net/de/kapitel/nummernprogrammekleinode-aus-aller-welt, 30.11.2014.

109 Die Autorin zitiert hier Kapitel 6 ihres Artikels: Moser, „Frühes Kino“, Kapitel 6 „Von der Ansicht zur Narration: Genres und Stars, http://ww1.habsburger.net/de/kapitel/von-der-ansicht-zurnarration-genres-und-stars, 30.11.2014.

110 Gunning, Non-Continous Style, S. 2. Jung, Uli: Ästhetischer Wandel. Von der ,Lebenden Photographie“ zum Filmgenre, in: Jung, Uli/Loiperdinger, Martin (Hg.): Geschichte des dokumentarischen Films in Deutschland, Bd. 1, Kaiserreich 1895-1918, Stuttgart 2005, S. 221-222. Gunning, Vor dem Dokumentarfilm, S. 114-117.

111 Gunning folgt hier der Genredefinition des Filmemachers John Grierson, der den Begriff „Dokumentarfilm“ etablierte. Vgl. dazu: Gunning, Vor dem Dokumentarfilm, S. 113, 117 f. Grierson, John: First Principles of Documentary, in: Hardy, Forsyth (Hg.), Grierson on Documentary, Berkley/Los Angeles 1966, S. 145-156. 
RIESENRAD. ${ }^{112}$ Von diesen frühesten Laufbildern der Residenzstadt Wien sind einige „Szenen“ erhalten geblieben. LE RING ${ }^{113}$ präsentiert das rege Treiben nahe der SirkEcke. ${ }^{114}$ Die Kamera ist ebenerdig gegenüber der Oper mit Einblick in die Kärntner Straße platziert. Die „Ansicht“ zeugt von einer speziellen Attraktivität. Der schräg angeschnittene Blickwinkel unterstreicht die Staffelungen des Bilds durch Laternenmasten und seitlich hervorspringende Häuserblöcke, die Raumtiefe vermitteln. Die Unterbrechung der Sicht durch dahineilende Passanten, Fiaker und Pferdestraßenbahnen, die im Vorder- und Hintergrund vorbeiziehen, wobei sich Kamera- und Handlungsachse laufend verschieben, wirkt dynamisierend. Direkt auf das Objektiv zusteuernde oder knapp passierende Personen und Fuhrwerke erzeugen ein Gefühl der unmittelbaren Anwesenheit. Die Kamera ist stellvertretend für den Zuschauer direkt im Geschehen, in beobachtender Position.

ENTRÉE DU CINEMATOGRAPHE ${ }^{115}$ nimmt den Ort der ersten kinematographischen Vorführungen in Wien (Kärntner Straße 45/Ecke Krugerstraße) in Augenschein. ${ }^{116}$ Die Aufnahmen sind von der gegenüberliegenden Straßenseite des Vorführlokals aufgenommen. Wiederum wurde eine leichte Querposition angenommen, um das Eckgebäude mit dem seitlich montierten Hinweisschild „Photographe, Cinématographe“ fassen zu können. Die Besucher des Etablissements werden zu kaum bestimmbaren Protagonisten in der Ferne - querende Kutschen verstellen laufend den Blick. Dominant sind die auf die Kamera zustrebenden Fußgänger. Sie drehen sich um, gehen nochmals vorbei, verweilen gebannt für Sekunden vor der technischen Neuheit oder weichen plötzlich wieder zurück. Wer einen Blick in die Kamera wagt, kann sein Konterfei bald selbst auf der Leinwand betrachten.

Die ersten Aufnahmen aus Wien ${ }^{117}$ entsprechen in der Konzeption durchaus den Lumière-Bildern aus anderen Städten der Welt. ${ }^{118}$ Ob es sich bei den jeweiligen Filmen um Lokalaufnahmen oder Städtebilder bzw. Reisebilder handelte, entschied

112 Kieninger, Wanderkinos, S. 57-58. Schauer, Peter: Zur Ur- und Frühgeschichte des österreichischen Films, Wien 1964, S. 13 und 17.

113 LE RING, F 1896, Produktion (P): Société Lumière.

114 Die Autorin zitiert hier Kapitel 5 ihres Artikels: Moser, „Frühes Kino“, Kapitel 5 „Nummernprogramme - Kleinode aus aller Welt, http://ww1.habsburger.net/de/kapitel/nummernprogrammekleinode-aus-aller-welt, 30.11.2014.

115 ENTRÉE DU CINEMTAOGRAPHE, F 1896, P: Société Lumière.

116 Die Autorin zitiert hier Kapitel 2 ihres Artikels: Moser, „Frühes Kino“, Kapitel 2 „Der erste Kinematograph in Wien - ein Medium erobert Österreich-Ungarn“, http://ww1.habsburger.net/de/kapitel/ der-erste-kinematograph-wien-ein-medium-erobert-oesterreich-ungarn, 30.11.2014.

117 Erhalten geblieben ist zudem die Szene RETOUR DES COURSES, F 1896, P: Société Lumière. Es handelt sich hierbei um das in den Quellen betitelte Filmdokument AUSFAHRT DER WIENER FIAKER.

118 Siehe dazu etwa die Ausführungen in: Jung, Uli: Städtebilder und Lokalaufnahmen, in: Jung, Uli/Loiperdinger, Martin (Hg.): Geschichte des dokumentarischen Films in Deutschland, Bd. 1, Kaiserreich 1895-1918, Stuttgart 2005, S. 282-287. Auch ein direkter Vergleich mit dem Bild [BUDAPEST], F 1896, P: Société Lumière, unterstützt diese Feststellung. 
letztlich der Auswertungskontext. Wurde eine Szene am „Drehort“ projiziert, war sie als lokal verortet anzusehen. Kam das Material auch an anderen örtlichen Schauplätzen zum Einsatz, konnte es als Städtefilm in das Nummernprogramm der Wanderunternehmer Eingang finden. ${ }^{119}$

Reisebilder erfreuten sich besonderer Popularität und offerierten ein ansprechendes Potpourri an Sehenswürdigkeiten, wie Parkanlagen, pittoreske Landschaften, Denkmäler, prunkvolle Gebäude, Stadtpanoramen, Jahrmarkteindrücke, exotische Tiere, fremde Sitten und Brauchtümer. Allgemeine Gesamtansichten wechselten zu belebten Straßen oder optisch interessanten Bauwerken. Die präsentierten „Ansichten“ griffen auf über Wochenillustrierte, Panoramen, Reisefotografien und Postkarten bereits konventionalisierte Ikonen zurück. ${ }^{120}$ Durch die Mobilisierung des Blicks ermöglichte die Kinematographie jedoch neue Formen der Wahrnehmung. Von erhöhten Standpunkten wurden Land- und Ortschaften in Panoramaschwenks abgelichtet. Noch deutlicher nachempfindbar machten die Reiseerfahrung allerdings die „phantom rides“: In Bewegung befindliche Fahrzeuge (Züge, Busse, Autos, Schiffe) dienten als mobile Aufnahmestandpunkte, die neue Formen der Landschaftsinszenierung und -erlebbarkeit ermöglichten. ${ }^{121}$

Der Kinematograph bot dem Publikum Ersatzreisen an. „In wenigen Minuten“ konnte man - laut werbender Presse - „unzählige Gegenden durcheilen“, „Ereignissen beiwohnen“, die man „niemals hätte sehen können“, um zugleich das persönliche „Wissen umfassend zu bereichern““. ${ }^{122}$ Auch traf der erste Höhepunkt der Kinematographie mit den Vorboten des Massentourismus zusammen. ${ }^{123}$ Vor allem entlang der Bahnlinien entwickelte sich ein reger Fremdenverkehrsstrom, der Sommerfrischler, Kurgäste und Wanderer zu den angestrebten Destinationen brachte. ${ }^{124}$ Schnell wurden die Werbemöglichkeiten der Reisebilder erkannt und genutzt. Die Präsentation der österreichisch-ungarischen Landschaften und Attraktionen oblag aber vorerst ausländischen Erzeugern. 1907 produzierte die britische Charles Urban Trading Co. mit Unterstützung des k.k. Eisenbahnministeriums das Bild ScHÖNHEITEN TIROLS. Eine Fahrtaufnahme sollte besondere Einblicke gewähren und zu einem Besuch der Region

119 Vgl. Kieninger, Wanderkinos, S. 295-297.

120 Ebd., S. 292. Jung, Städtebilder, S. 293. Deeken, Annette: Geschichte und Ästhetik des Reisefilms, in: Jung, Uli/Loiperdinger, Martin (Hg.): Geschichte des dokumentarischen Films in Deutschland, Bd. 1, Kaiserreich 1895-1918, Stuttgart 2005, S. 299-300, 309. Garncarz, Der nicht-fiktionale Film im Programm der Wanderkinos, S. 119.

121 Kieninger, Wanderkinos, S. 292.

122 Österreichischer Komet, „Der Kinematograph als Ersatz für Reisen““”, Nr. 5, 1. November 1908, S. 5 .

123 Die Autorin zitiert hier Kapitel 4 ihres Artikels: Moser, Filmischer Aufbruch in die Moderne, Kapitel 4 „Vom Fahren und Reisen: Fremdenverkehr und Tourismusfilme“, http://ww1.habsburger. net/de/kapitel/vom-fahren-und-reisen-fremdenverkehr-und-tourismusfilme, 30.11.2014.

124 Sandgruber, Ökonomie und Politik, S. 284-287. 
anregen. ${ }^{125}$ Im Jahr 1908 gab ferner der „Ausschuss des Niederösterreichischen Landesverbandes für Fremdenverkehr“ bekannt, künftig „kinematographische Aufnahmen zur Hebung des Tourismusgewerbes“ $\mathrm{zu}$ forcieren. ${ }^{126}$

Bilder aus der Habsburgermonarchie wurden speziell außerhalb des Landes gerne dargeboten. Ludwig Neumayers „Erste Bayerische Filmfabrik“ in Straubing hatte unter anderem Filme aus dem österreichischen Salzkammergut im Repertoire (z. B. HAllSTATt UND DER HALlStÄTtER SEE, D 1910), ${ }^{127}$ die „Internationale Kinematographengesellschaft Berlin“ vertrieb das Bild REISE DURCH TIROL (D 1902) ${ }^{128}$ und auch „Reisebilder“ aus Wien waren stets beliebt und wurden u. a. von der französischen „Pathé“ offeriert. ${ }^{129}$

Eine durchaus werbende Wirkung ist mitunter auch den frühen Industriebildern zuzuschreiben. Bereits um 1900 wurden in Frankreich und in den USA erste industrielle Auftragsfilme für Unternehmen angefertigt. In den seltensten Fällen können jedoch in der Frühphase bis 1910 die Herstellungsbedingungen dieser Aufnahmen, und somit die ursprünglichen Impulsgeber (Filmproduzenten oder Fabrikanten), eruiert werden. ${ }^{130}$

Ab 1907 etabliert sich in den kinematographischen Fachblättern und Programmen die Bezeichnung „Industriebild“ für Filme, die den produktionstechnischen Werdegang von Konsumgütern, Nahrungsmitteln und bisweilen Luxuswaren detailliert beschrieben. Diese Filme präsentieren idealisierte Abläufe. Eifrige und disziplinierte Arbeiterinnen und Arbeiter gehen, im Bewusstsein, unter permanenter Beobachtung zu stehen, ihrer Tätigkeit nach. Immer wieder sind die „Darsteller“ auch Teil einer folkloristischen Inszenierung, wenn sie sich etwa als Repräsentanten des ortsüblichen Brauchtums in ihrer Tracht, ihrem Gestus oder ihrer Motorik zu erkennen geben. Hier verschwimmen die Grenzen zwischen Reise- und Industriebild, was sich auch an der Titelgebung (z. B. FABRIKATION VENEZIANISCHER SPITZEN, FäCHERINDUSTRIE IN JAPAN, HERSTELLUNG VON HOLLÄNDISCHEM KäSE) erkennen lässt. ${ }^{131}$ Doch während in den ländlichen und städtischen Ansichten vor allem räumliche

125 Kieninger, Wanderkinos, S. 294-295. Kinematographische Rundschau, Nr. 9, 1. Juni 1907, S. 4.

126 Kinematographische Rundschau, Nr. 37, 1. August 1908, S. 5.

127 Deeken, Geschichte und Ästhetik des Reisefilms, S. 314.

128 Kieninger, Wanderkinos, S. 427.

1291910 wird das Bild WIEN UND SEINE HAUPT-SEHENSWÜRDIGKEITEN (Der Opernplatz, Parlamentsgebäude und Rathaus, Eingang zur Hoch- und Untergrundbahn und Karlskirche, Eingang zum Prater, Stefanskirche, Denkmal der Kaiserin Elisabeth, Die Rotunde) angeboten: Österreichischer Komet, „Neue Filme“, Nr. 56, 26. November 1910, S. 8. Auch eine Verleihanstalt bietet Ende 1909 eine Naturaufnahme aus Wien an: Österreichischer Komet, „Anzeige Projektograph Kinematographen- und Filmfabrik“, Nr. 31, 7. Dezember 1909, S. 12.

130 Zimmermann, Yvonne: Industriefilme, in: Zimmermann, Yvonne (Hg.): Schaufenster Schweiz. Dokumentarische Gebrauchsfilme 1896-1964, Zürich 2011, S. 253 und 266.

131 Loiperdinger, Martin: Industriebilder, in: Jung, Uli/Loiperdinger, Martin (Hg.): Geschichte des dokumentarischen Films in Deutschland, Bd. 1, Kaiserreich 1895-1918, Stuttgart 2005, S. 325-327, 332. 
Organisationsprinzipien zum Tragen kommen, beruht die Montage bei den industriellen Aufnahmen vornehmlich auf zeitlichen Abläufen: Die einzelnen Herstellungsschritte werden chronologisch aneinandergereiht. ${ }^{132}$ Halbnahe und nahe Einstellungen erlaubten eine genaue optische Untersuchung des gezeigten Prozesses und wurden schnell zu einem standardisierten Stilmittel des industriellen Bilds. Im Streben nach dem größeren filmischen Effekt entwickelte sich demnach die Filmsprache fast „nebenbei“. 133

Die ersten Industriefilme österreichischer Provenienz stammen aus den 1910erJahren ${ }^{134}$ und somit aus einer Zeit, als „Naturaufnahmen“ auch in ortsfesten Kinos bereits zu einem fixen Programmpunkt zählten, allerdings nicht mehr im Bereich der Hauptattraktionen, sondern als Begleitbilder zum Langfilm. Nach und nach hatte sich die Laufzeit der präsentierten Laufbilder verlängert: Um 1907 wiesen die im Kino vorgeführten Filme eine durchschnittliche Länge von 6 Minuten auf. 1909 lag die Projektionszeit bei vielen Filmen bereits bei über 20 Minuten, der Mittelwert belief sich auf 8 Minuten und 50 Sekunden. ${ }^{135}$ Nicht-fiktionale Filme waren um 1900 zwischen ein und vier Minuten lang, um 1914 lag dieser Wert bei sieben bis zwölf Minuten. ${ }^{136}$ Parallel dazu nahm der Anteil der fiktionalen Filme laufend zu, ab 1906 dominierten sie mit einer Zweidrittelmehrheit das Gesamtangebot. Sämtliche anerkannte Hersteller boten nun keine „Bilder“ mehr an, sondern offerierten Dramen, Komödien, Lustspiele oder Tragödien und orientierten sich folglich an klassischen Kategorien. ${ }^{137}$

Zudem veränderte sich die Aufführungspraxis - statt in Wanderkinos wurde zunehmend in festen Etablissements gespielt. ${ }^{138}$ In Wien lassen sich die ersten permanenten Lichtspieltheater in den Jahren 1903 bis 1905 nachweisen. 1908 erfolgte die Gründung des „Reichsverbandes der Kinematographenbesitzer“, in dem die

132 Gunning, Vor dem Dokumentarfilm, S. 115-117.

133 So zitiert nach: Kieninger, Wanderkinos, S. 146, 222.

134 Einzig zwei Hinweise auf Frühformen des Industriefilms finden sich vor 1902: DAS EISEN (A 1902, Produkton: Friedrich Umlauft) sowie DER MAGEN von Wien (A 1902, P: Max Winter), ein Film über den Ablauf der Fleischerzeugung. Ob es sich beim Hersteller des Films DER MAGEN voN WIEN, um den für seine Sozialreportagen bekannten Journalisten und späteren sozialdemokratischen Politiker, Max Winter handelte, konnte nicht geklärt werden. Vgl. dazu: Kieninger, Wanderkinos, S. 299 bzw. Znaimer Wochenblatt, Nr. 28, 9. April 1902, S. 7.

135 Hier wird den Berechnungen der Studie von Ernst Kieninger gefolgt: Ebd., S. 315-316.

136 Jacques, Pierre-Emmanuel/Zimmermann, Yvonne: Dokumentarischer Film in der Schweiz im historischen Überblick (1896-1964), in: Zimmermann, Yvonne (Hg.): Schaufenster Schweiz. Dokumentarische Gebrauchsfilme 1896-1964, Zürich 2011, S. 93.

137 Kieninger, Wanderkinos, S. 315-316, 325. Jacques und Zimmermann setzen den Zeitpunkt der zunehmenden Dominanz des abendfüllenden Spielfilms ab 1911 an. Jacques/Zimmermann, Dokumentarischer Film, S. 96.

138 Die Autorin zitiert hier Kapitel 3 ihres Artikels: Moser, „Frühes Kino“, Kapitel 3 „Vom Schaustellerbetrieb zum Kinotheater“, http://ww1.habsburger.net/de/kapitel/vom-schaustellerbetriebzum-kinotheater, 30.11.2014. 
Wanderkinounternehmer allerdings klar unterrepräsentiert waren. Auch nahmen die Betreiber ortsfester Einrichtungen ihren Kollegen aus dem Schaubudenmilieu gegenüber verstärkt eine ablehnende Haltung ein. Sie waren bestrebt, das Ansehen ihres Metiers über eine Distanzierung vom Jahrmarktbudenbetrieb, hin zu einer Annäherung an das „künstlerisch hochwertig“ eingeschätzte Theater zu heben. ${ }^{139}$ 1909 existierten in der k.k. Residenzstadt bereits 62 ständige Kinos gegenüber 14 Wandereinrichtungen. 1913 zählte man im Gesamtgebiet der Habsburgermonarchie 400 kinematographische Betriebe, davon entfielen 200 auf Wanderunternehmer. In Wien konnte man in diesem Jahr 130 permanente Lichtspieltheater statistisch erfassen. $^{140}$

139 Schwarz, Kino und Kinos, S. 12, 22.

140 Ebd., S. 22-23. Kino-Journal, Nr. 161, 14.6.1913, S. 4-5. Polizeiarchiv, Bundespolizeidirektion Wien, Karton 1, „Kinozensur in Wien“, Fremden-Blatt, 11.5.1913, o. S. 